\title{
A New Method to Estimate the Magnetic Field Modulation Effect of Brushless Doubly-Fed Machine with Cage Rotor
}

\author{
Hanghang Liu *, Li Han **, Qiang Gao ***
}

\begin{abstract}
Brushless doubly-fed machine (BDFM) doesn't use brush and slip ring, and has advantages such as high system reliability, small capacity of its frequency converter, low system cost, adjustable power factor and speed, etc. At the same time, it has good applicable potentials on the variable frequency motors and the variable speed constant frequency generators. However, due to the complicacy and particularity of BDFM in the structure and operating mechanism, the effect of magnetic field modulation directly influences the operating efficiency of BDFM. To study the effect of different cage rotor structures on the magnetic field modulation of BDFM, the rotor magnetomotive force (MMF) of BDFM with cage rotor is studied by the analytical method. The components and features of rotor harmonic MMFs are discussed. At the same time, the method to weaken the higher harmonics is analyzed by the theoretic formulae. Furthermore, the magnetic field modulation mechanism is expounded on in detail and the relationship between the magnetic field modulation effect and the operating efficiency of BDFM is established. And then, a new method for estimating the magnetic field modulation effect is proposed. At last, the magnetic field modulation effects of four BDFM prototypes with different cage rotor structures are compared by the MMF analysis and the efficiency data of electromagnetic design. The results verify the effectiveness of the new method for estimating the magnetic field modulation effect of BDFM with cage rotor.
\end{abstract}

Keywords: BDFM, Magnetic Field modulation, Operating efficiency

\section{Introduction}

The Brushless Doubly-Fed Machine (BDFM) possesses similar performance to both induction and synchronous motors, and has a wide range of applications, especially in the frequency-control driving system and variable-speed constant-frequency power-generating system [1], [2]. Compared with the ordinary AC machines, however, the structure and the operating mechanism of BDFM are rather special and complex. BDFM has two sets of stator windings, namely power winding (PW) and control winding (CW). The PW and the $\mathrm{CW}$ do not have direct electromagnetic coupling and the energy conversion between the mechanical power and the electric power depends on the magnetic field modulation of the special rotor structure. It is necessary to study the rotor structure and the magnetic field modulation effect because of its crucial function on energy conversion of

* Shandong Dongying Power Supply Company, Dongying, China. (liuhang701@gmail.com)

** State Key Laboratory of Power Transmission Equipment \& System Security and New Technology, Chongqing University, China. (hanli@cqu.edu.cn)

*** Zhejiang Taizhou Power Supply Company, Taizhou, China (gaoqiang242@163.com)

Received 24 May 2012; Accepted 02 August 2012
BDFM. A general way to estimate the rotor magnetic field modulation effect is to use the electromagnetic field numerical calculation by finite element method (FEM), and then analyze the harmonic spectrum of the air gap magnetic field [3]-[7]. However, FEM needs complicated numerical calculations and is timeconsuming.

As the sister paper of [3], this paper develops the rotor MMF analysis of BDFM with cage rotor by the analytical method. A new method for estimating the rotor magnetic field modulation effect without FEM analysis is proposed. And then, the magnetic field modulation effects of four BDFM prototypes with different cage rotor structures are compared by the new method and the validity of the new method is illustrated.

\section{The Basic Structure of BDFM}

The basic structure of BDFM system is shown in Fig. 1. Two sets of 3-phase AC windings, the PW with the number of pole-pairs $\mathrm{pp}$ and the $\mathrm{CW}$ with the number of pole-pairs pc, are embedded in the stator core. The 
PW is connected to 3-phase grid directly and has a constant frequency $\mathrm{fp}$. The $\mathrm{CW}$ is connected to a bidirectional converter and has an adjustable frequency fc [3].

BDFM has three main rotor structures which are salient reluctance rotor, cage rotor and axially laminated anisotropic rotor. The most commonly used structure is cage rotor. This paper focuses on four different cage rotor structures, shown in Fig. 2. Model I is the cage rotor with common bar, Model II is the cage rotor with common bar and common end ring, Model III is the cage rotor without common bar and Model IV is the cage rotor with equidistant bar span [7]. There is no external power source on the cage rotor and the rotor bars are shorted.

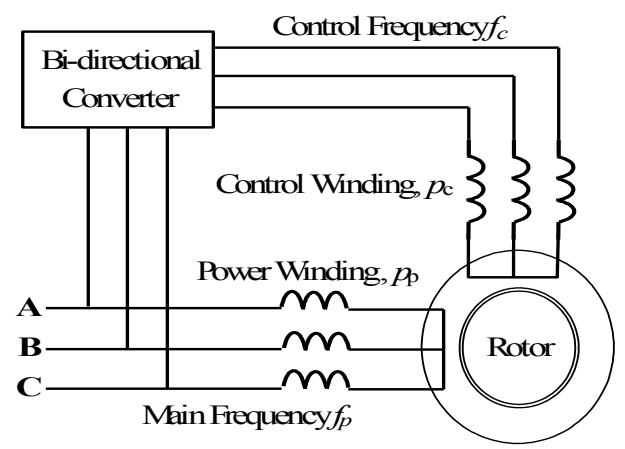

Fig. 1. The structure of BDFM

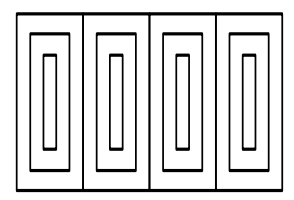

(a) Model I

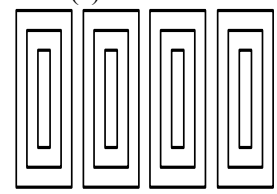

(c) Model III
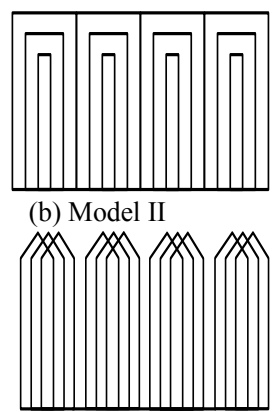

(d) Model IV
Fig. 2. The structures of cage rotors

The number of rotor nests is equal to the number of rotor pole pairs $\mathrm{pr}(\mathrm{pr}=\mathrm{pp}+\mathrm{pc})$. The number of shortcircuit loops within each nest is Nr. All short circuit loops of Models I-III are concentric and the outermost short-circuit loop shares a common cage bar with the nearby nest for Model I and Model II. However, each short-circuit loop is evenly distributed and has the same span for Model IV.

\section{Field Modulation Mechanism of BDFM}

A series of stator odd-harmonic MMFs are induced when 3-phase sinusoidal currents with different frequencies flow through the $\mathrm{PW}$ and the $\mathrm{CW}$. The harmonics of the air-gap magnetic flux density induce rotor EMF and rotor current. Suppose the number of rotor nests is $\mathrm{x}(1 \leq \mathrm{x} \leq \mathrm{pr})$ and the number of shortcircuit loops in each nest is $n(1 \leq n \leq M)$, as shown in Fig. 3. The rotor current, which is induced by the PW's vth harmonic air-gap magnetic flux density, in the nth short-circuit loop of the xth nest is as follows [3]:

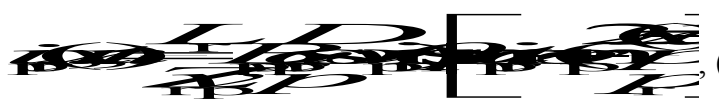

where $\mathrm{L}$ are Dr are the length and the outer diameter of the rotor core, respectively, $\mathrm{Zr}$ is the impedance of a short-circuit loop, $\mathrm{v}$ is the harmonic order of the airgap magnetic flux density, Bpv is the vth harmonic airgap magnetic flux density produced by the $\mathrm{PW}$, wpvr is the angular frequency of the rotor EMF induced by Bpv, and $\theta \mathrm{n}$ is the span angle between the bar of the $\mathrm{nth}$ short-circuit loop and the center axis of the shortcircuit loop within a nest.

Define the amplitude, $\operatorname{Ipvr}(n)$, of $\operatorname{ipvr}(x, n, t)$ as
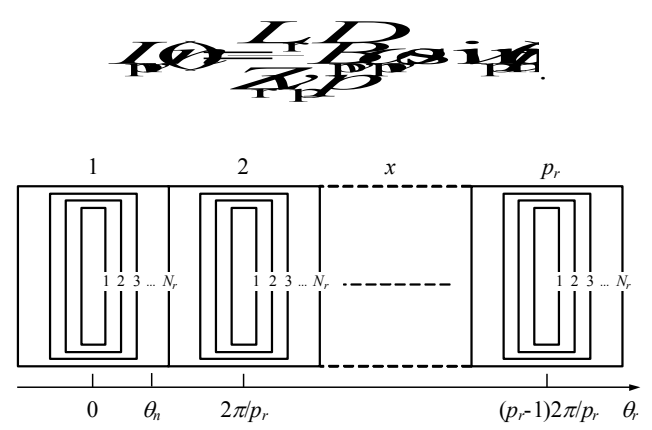

Fig. 3. The numbering of nests and short-circuit loops of Model I

Equation (1) can then be simplified as

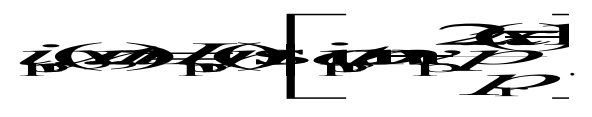

The amplitude of the rotor current ipvr is a function of the short-circuit loop number $\mathrm{n}$ while the other parameters remain constant. In other words, the amplitudes of the rotor currents in the different shortcircuit loops of the same nest are different.

In order to obtain the MMF of the rotor short-circuit loop, firstly the vth harmonic MMF can be expressed as follows: 


$$
f=\frac{14 \pi}{2 \pi 2} \text { Cot }
$$

where $\mathrm{N}$ and $\mathrm{i}$ are the turns and the current of the coil, respectively, and $\theta$ is the space angle.

Each short-circuit loop of the cage rotor can be considered as a single-turn coil, so that $\mathrm{v}=\mathrm{vpr}, \mathrm{i}=\mathrm{ipvr}$, $\theta=\theta \mathrm{r}-2(\mathrm{x}-1) / \mathrm{pr}$, and $\mathrm{N}=1 \times \mathrm{kvpr}, \quad$ in which $\mathrm{kvpr}=\sin (v \operatorname{vpr} \theta \mathrm{n})$ is the vprth harmonic pitch factor of the short-circuit loop.

According to (3), (4) and the analysis mentioned above, the harmonic MMF in one rotor short-circuit loop, produced by the PW's vth harmonic air-gap magnetic flux density, can then be expressed as

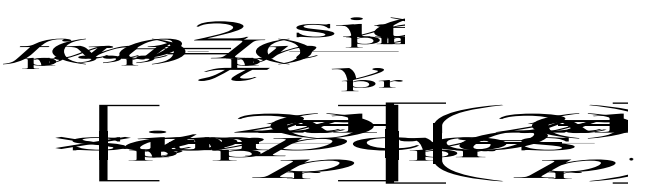

where $\mathrm{vpr}=1,3,5, \ldots$ are the harmonic orders of the rotor MMF.

Equation (5) shows that the harmonic MMF of one rotor short-circuit loop is a plant MMF, which distributes co-sinusoidal on space and varies sinusoidal in time.

It follows that the total rotor harmonic MMF, induced by the PW's vth harmonic air-gap magnetic flux density, can be obtained as:

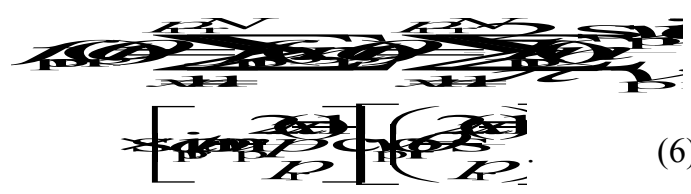

Similar to (6), the total rotor harmonic MMF, induced by the CW's vth harmonic air-gap magnetic flux density, is:

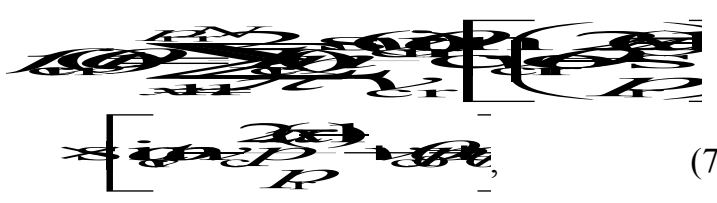

where Icvr is the amplitude of the rotor current induced by the CW's vth harmonic air-gap magnetic flux density, $\omega c v r$ is the angular frequency of Icvr, $\theta 0$ is the space angle between the $\mathrm{PW}$ axis and the $\mathrm{CW}$ axis, and $\varphi 0$ is the time phase angle between the PW current and the $\mathrm{CW}$ current.

Suppose $\mathrm{pp}=3, \mathrm{pc}=1, \mathrm{pr}=4$ and $\mathrm{v}=\mathrm{vpr}=\mathrm{vcr}$. Then, (6) and (7) can be then simplified as follows, respectively:

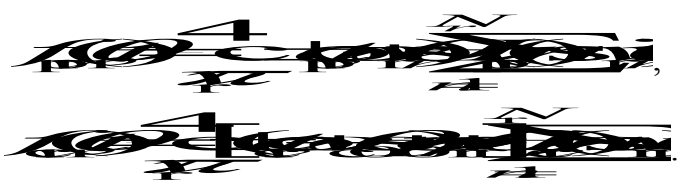

when $\mathrm{vr}=4 \mathrm{k}+1 \quad(\mathrm{k}=0,1,2,3, \ldots)$, the sign before $\omega \mathrm{pvr}$ is positive in (8), which represents the backward-rotating MMFs; and the sign before $(\omega \mathrm{cvrt}+v p c \theta 0+\varphi 0)$ is negative in (9), which represents the forward-rotating MMFs. When $\mathrm{vr}=4 \mathrm{k}-1 \quad(\mathrm{k}=1,2,3, \ldots)$, the sign before wpvr is negative in (8), which represents the forwardrotating MMFs; and the sign before $(\omega \mathrm{cvrt}+\mathrm{vpc} \theta 0+\varphi 0)$ is negative in (9), which represents the backwardrotating MMFs.

Assume Fpvr and Fcvr are as follows, respectively

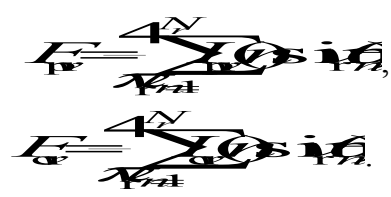

Equation (8) and (9) can then be expressed as follows, respectively

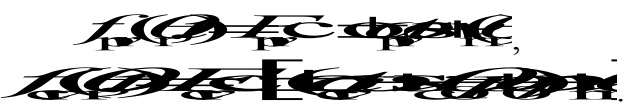

The tidy and clear expressions of the rotor harmonic MMFs are obtained by (12) and (13) at last. While there are abundant rotor harmonics in which ppth and pcth harmonics (**This sentence does not seem to make sense**) are beneficial to enhance the magnetic field modulation effect of BDFM with cage rotor, other harmonics are harmful and should be weakened as much as possible.

\section{Analysis and Calculations}

To study the rotor MMFs and the magnetic field modulation mechanism, the vrth harmonic pitch factor, $\mathrm{kvr}$, of the rotor short-circuit loop is rewritten as follows:

$$
k_{v \mathrm{r}}=\sin \left(v_{\mathrm{r}} \theta_{\mathrm{n}}\right) \text {. }
$$

From (10) and (11), it can be seen that the amplitudes of the rotor harmonic MMFs are determined by $\mathrm{kvr}$ when other parameters remain constant. However, kvr is dependent on the order of harmonics vr and the span angle $\theta$ n.

For Model I and Model II shown in Fig. 1, the span 
angle of the various short-circuit loops is

$$
Q_{\mathrm{n}}=\frac{(\boldsymbol{2}-1)}{P_{\mathrm{r}}(2 \mathrm{Y}-1}
$$

where $n=1,2,3, \ldots, N_{\mathrm{r}}$.

For Model III shown in Fig. 1, the span angle of the various short-circuit loops is changed to

$$
\theta_{\mathrm{n}}=\frac{(2 n-1) \pi}{2 p_{\mathrm{r}} Y}
$$

For Model IV shown in Fig. 1, the span angle of each short-circuit loop is the same, that is, it is independent of the numbering of the short-circuit loop $\mathrm{n}$.

According to (10), (11) and (14), it is easily understood that the rotor 5th harmonic MMF can be eliminated when $\theta n=\pi / 5$, the rotor 7 th harmonic MMF can be eliminated when $\theta n=\pi / 7$, the rotor 9th harmonic MMF can be eliminated when $\theta n=\pi / 9$, and so on. To reduce the harmful odd harmonics, the span angles of the concentric short-circuit loops in Models I, II and III should be set around $\pi / 5, \pi / 7, \pi / 9$, etc.

To analyze and calculate the rotor magnetic field modulation effects, the common parameters of the four BDFM prototypes are listed in Table 1.

Table 1. Common parameters of the BDFM prototypes

\begin{tabular}{|c|c|}
\hline Parameter & Value \\
\hline Outer diameter of the stator $D_{1}$ & $260 \mathrm{~mm}$ \\
\hline Inner diameter of the stator $D_{i 1}$ & $170 \mathrm{~mm}$ \\
\hline Outer diameter of the rotor $D_{2}$ & $168.9 \mathrm{~mm}$ \\
\hline Inner diameter of the rotor $D_{i 2}$ & $60 \mathrm{~mm}$ \\
\hline Length of the iron core $L$ & $120 \mathrm{~mm}$ \\
\hline Number of PW pole-pairs $p_{p}$ & 3 \\
\hline Number of CW pole-pairs $p_{c}$ & 1 \\
\hline Number of stator slots $Q_{s}$ & 36 \\
\hline Number of rotor nests $p_{r}$ & 4 \\
\hline $\begin{array}{c}\text { Number of short-circuit loops } \\
\text { in a nest } N_{r}\end{array}$ & 6 \\
\hline
\end{tabular}

For Model I, Model II and Model III, the harmonic pitch factors of the various rotor short-circuit loops are calculated by (14) and listed in Table II and Table III.

For the concentric short-circuit loop structures of Model I, Model II and Model III illustrated in Fig. 1, Table II and Table III show that the rotor MMF pitch factors of the outer short-circuit loops $(n=4,5,6)$ are larger than those of the inner short-circuit loops $(n=1,2,3)$. The result indicates that the outer shortcircuit loops in the nest play a more important role to enhance the beneficial 1 st and 3rd harmonics and improve the magnetic field modulation effect. At the same time, the rotor MMF pitch factors of Model I (the cage rotor with common bar) and Model II (the cage rotor with common bar and common end ring) are the same, and they are larger than those of Model III (the cage rotor without common bar). The result indicates that the rotor magnetic field modulation effects of Model I and Model II are equal but better than those of Model III. The validity of the results mentioned above is coincident with [3], [6]. On the other hand, each rotor MMF pitch factor and the magnetic field modulation effect of the various short-circuit loops in Model IV (the cage rotor with equidistant bar spans) are equal due to having the same span angle.

To compare the magnetic field modulation effects of Models I-IV and avoid the complicated FEM numerical computation and harmonic spectrum analysis in [3], a new method is proposed as follows in this paper. The essence of the magnetic field modulation mechanism of BDFM can be reflected by the efficiency of the electromechanical energy conversion. The rotor of a BDFM plays the key function in the magnetic field modulation and the energy conversion. If the magnetic field contains more beneficial 1 st and $3 \mathrm{rd}$ harmonics and less harmful harmonics, the efficiency of the electro-mechanical energy conversion is higher and the magnetic field modulation effect is better. To obtain the operating efficiency of BDFM with the various cage rotor structures directly, a software program for BDFM electromagnetic design, based on the magnetic circuit analysis, is programmed on the platform of Visual Basic 6.0. Compared with FEM, the new idea mentioned above comes from the physical concept of the magnetic field modulation mechanism of the BDFM, which is more valid, intuitive and timesaving.

Table 2. Rotor MMF pitch factors of Model I and

\begin{tabular}{|c|c|c|c|c|c|}
\hline \multirow{2}{*}{$\begin{array}{l}\text { Loop } \\
\text { numb } \\
\text { ering }\end{array}$} & \multirow{2}{*}{$\begin{array}{l}\text { Span } \\
\text { angle }\end{array}$} & \multicolumn{2}{|c|}{$1^{\text {st }}$ harmonic } & \multicolumn{2}{|c|}{$3^{\text {rd }}$ harmonic } \\
\hline & & PW & $\mathrm{CW}$ & PW & $\mathrm{CW}$ \\
\hline 1 & $4.09^{\circ}$ & 0.0152 & 0.0051 & 0.0452 & 0.015 \\
\hline 2 & $12.3^{\circ}$ & 0.1274 & 0.0452 & 0.3591 & $\begin{array}{c}0.127 \\
4\end{array}$ \\
\hline 3 & $20.5^{\circ}$ & 0.3067 & 0.1221 & 0.7703 & $\begin{array}{c}0.306 \\
7\end{array}$ \\
\hline 4 & $28.6^{\circ}$ & 0.4780 & 0.2297 & 0.9949 & $\begin{array}{c}0.478 \\
0\end{array}$ \\
\hline 5 & $36.8^{\circ}$ & 0.5615 & 0.3591 & 0.8779 & $\begin{array}{c}0.561 \\
5\end{array}$ \\
\hline 6 & $45.0^{\circ}$ & 0.5000 & 0.5000 & 0.5000 & $\begin{array}{c}0.500 \\
0\end{array}$ \\
\hline
\end{tabular}
Model II 
Table3. Rotor MMF pitch factors of Model III

\begin{tabular}{|c|c|c|c|c|c|}
\hline $\begin{array}{c}\text { Loop } \\
\text { numberin } \\
\mathrm{g}\end{array}$ & \multirow{2}{*}{$\begin{array}{c}\text { Span } \\
\text { angle }\end{array}$} & \multicolumn{2}{|c|}{$1^{\text {st }}$ harmonic } & \multicolumn{2}{|c|}{$3^{\text {rd }}$ harmonic } \\
\cline { 3 - 6 } & $4.09^{\circ}$ & $\begin{array}{c}0.012 \\
8\end{array}$ & $\begin{array}{c}0.004 \\
3\end{array}$ & $\begin{array}{c}0.038 \\
1\end{array}$ & 0.0128 \\
\hline 2 & $12.3^{\circ}$ & $\begin{array}{c}0.108 \\
4\end{array}$ & $\begin{array}{c}0.038 \\
1\end{array}$ & $\begin{array}{c}0.308 \\
7\end{array}$ & 0.1084 \\
\hline 3 & $20.5^{\circ}$ & $\begin{array}{c}0.267 \\
3\end{array}$ & $\begin{array}{c}0.103 \\
3\end{array}$ & $\begin{array}{c}0.691 \\
3\end{array}$ & 0.2673 \\
\hline 4 & $28.6^{\circ}$ & $\begin{array}{c}0.433 \\
8\end{array}$ & $\begin{array}{c}0.195 \\
6\end{array}$ & $\begin{array}{c}0.961 \\
9\end{array}$ & 0.4338 \\
\hline 5 & $36.8^{\circ}$ & $\begin{array}{c}0.544 \\
9\end{array}$ & $\begin{array}{c}0.308 \\
7\end{array}$ & $\begin{array}{c}0.961 \\
9\end{array}$ & 0.5449 \\
\hline 6 & $45.0^{\circ}$ & $\begin{array}{c}0.548 \\
2\end{array}$ & $\begin{array}{c}0.434 \\
7\end{array}$ & $\begin{array}{c}0.691 \\
3\end{array}$ & 0.5482 \\
\hline
\end{tabular}

According to the electromagnetic design software of BDFM and keeping the output power constant at $4.33 \mathrm{~kW}$, the parameters such as air-gap flux density, electromagnetic torque, input power, losses and efficiency of four BDFM prototypes operating at the maximum state $(\mathrm{Uc}=380 \mathrm{~V}$ and $\mathrm{fc}=50 \mathrm{~Hz})$ are calculated and listed in Table IV. The results show that Model I and Model II have the highest efficiency and then the best magnetic field modulation effect, Model IV is almost as the same as Model I and Model II, and Model III has the lowest efficiency and the worst magnetic field modulation effect.

Table 4. Comparison of electromagnetic design data for 4 BDFM prototypes

\begin{tabular}{|c|c|c|c|}
\hline Parameter & $\begin{array}{c}\text { Models } \\
\text { I and II }\end{array}$ & $\begin{array}{c}\text { Model } \\
\text { III }\end{array}$ & $\begin{array}{c}\text { Model } \\
\text { IV }\end{array}$ \\
\hline PW voltage (V) & 380 & 380 & 380 \\
\hline PW frequency (Hz) & 50 & 50 & 50 \\
\hline CW voltage range (V) & $0 \sim 380$ & $0 \sim 380$ & $0 \sim 380$ \\
\hline CW frequency range & $-50 \sim 50$ & $-50 \sim 50$ & $-50 \sim 50$ \\
\hline Number of rotor slots & 44 & 48 & 44 \\
\hline $\begin{array}{c}\text { Air-gap flux density } \\
(\mathrm{T})\end{array}$ & 0.68 & 0.67 & 0.68 \\
\hline Line load of PW (A/m) & 1256 & 1600 & 1254 \\
\hline Line load of CW (A/m) & 755 & 954 & 756 \\
\hline $\begin{array}{c}\text { Current density of PW } \\
\left(\mathrm{A} / \text { mm }^{2}\right)\end{array}$ & 4.56 & 5.81 & 4.55
\end{tabular}

\begin{tabular}{|c|c|c|c|}
\hline $\begin{array}{c}\text { Stator and rotor copper } \\
\text { losses }(\mathrm{kW})\end{array}$ & 0.95 & 1.59 & 0.95 \\
\hline $\begin{array}{c}\text { Stator and rotor iron } \\
\text { losses (kW) }\end{array}$ & 0.71 & 0.40 & 0.72 \\
\hline $\begin{array}{c}\text { Mechanical and stray } \\
\text { losses (kW) }\end{array}$ & 0.11 & 0.11 & 0.11 \\
\hline $\begin{array}{c}\text { Electromagnetic torque } \\
\text { (N.m) }\end{array}$ & 27.56 & 27.57 & 27.59 \\
\hline Efficiency (\%) & 71.02 & 67.37 & 70.99 \\
\hline
\end{tabular}

\section{Conclusion}

A series of odd-harmonic MMFs occur in the cage rotor of BDFM when the currents of the rotor shortcircuit loops are induced by the air-gap magnetic field corresponding to the stator PW and CW currents. The rotor 1 st and 3rd harmonic MMFs are beneficial to enhance the operating efficiency and the magnetic field modulation effect of BDFM. The rotor's other higherorder harmonic MMFs are harmful and should be weakened as much as possible. The magnetic field modulation effect and the operating efficiency of BDFM with cage rotor have an inherent relationship. The relationship between the magnetic field modulation effect and the operating efficiency of BDFM with cage rotor is built up by the physical concept of electro-mechanical energy conversion and the magnetic field modulation mechanism. A new method to estimate the magnetic field modulation effect, which is more effective, intuitive, time-saving and can be applied to other different BDFM structures, was proposed. Reasonable distribution of short-circuit loops is a good way to weaken the harmful harmonics and improve the magnetic field modulation effect and efficiency of BDFM with cage rotor

\section{References}

[1] McMahon Richard A., Wan Xiaoyan, Abdi-Jalebi Eshan, Tavner Peter J., Roberts Paul C., Jagiela Mariusz, "The BDFM as a Generator in Wind Turbines," 12th International Power Electronics and Motion Control Conference, Portoroz, Aug. 30-Sept. 1 2006, pp. 18591865.

[2] McMahon R. A., Roberts P. C., Wang X., Tavner P. J., "Performance of BDFM as Generator and Motor," IEEE Proceedings-Electric Power Applications, vol. 153, no. 2, pp. 289-299, Mar. 2006.

[3] Qiang Gao, Li Han, Ci-yong Luo, Ren-jie Ye, "Cage Rotor Structure and Its Effect on Magnetic Field Modulation of Brushless Doubly-Fed Machine," Proceedings of the 11th International Conference on Electrical Machines and Systems, Oct. 17-20 2008, pp. 4234-4239. 
[4] Fengge Zhang, Guangjian Pan, Fengxiang Wang, Ming Lin, "Study on Design of Brushless Doubly-Fed Machine with a New Type Rotor," IEEE International Conference on Industrial Technology, Feb. 2009, pp. 1-3.

[5] Fengge Zhang, Yongxin Li, Xiuping Wang, "The Design and FEA of Brushless Doubly-fed Machine with Hybrid Rotor," Proceedings of 2009 IEEE International Conference on Applied Superconductivity and Electromagnetic Devices, Sept. 25-27 2009, pp. 324-327.

[6] Feng-xiang Wang, Feng-ge Zhang, "Brushless DoublyFed AC Machine with Magnetic Field Modulation Type," Changchun: Jilin University Press, 2004, pp. 1823.

[7] Xian-ming Deng, Rong-hui Fang, etc. et al., "Finite Element Analysis of Brushless Doubly-fed Machine with Equidistant Cage Rotor," Electric Machines and Control, vol. 13, no. 4, pp. 507-510, 522, Jul. 2009.

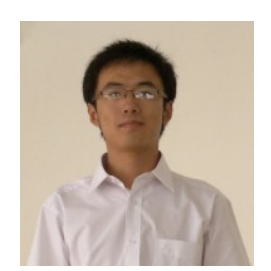

Hang-hang Liu received Master's degree in Electrical Engineering from Chongqing University. His research interests are emerging power systems and electric machines.

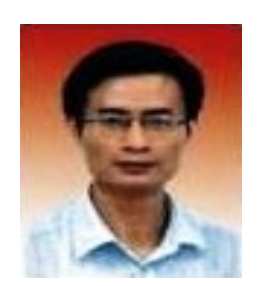

Li Han received Doctor's degree in Electrical Engineering from Chongqing University. His research interests are wind power technology and permanent magnet motors.

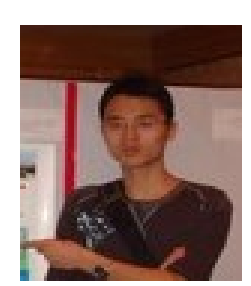

Qiang Gao received Master's degree in Electrical Engineering from Chongqing University. His research interests are emerging power systems and electric machines. 